\title{
Téoros
}

Revue de recherche en tourisme

\section{Tourisme et économie internationale}

\section{François Vellas}

Volume 8, numéro 2, juillet 1989

URI : https://id.erudit.org/iderudit/1080327ar

DOI : https://doi.org/10.7202/1080327ar

Aller au sommaire du numéro

\section{Éditeur(s)}

Université du Québec à Montréal

\section{ISSN}

0712-8657 (imprimé)

1923-2705 (numérique)

Découvrir la revue

Citer cet article

Vellas, F. (1989). Tourisme et économie internationale. Téoros, 8(2), 36-39.

https://doi.org/10.7202/1080327ar d'utilisation que vous pouvez consulter en ligne.

https://apropos.erudit.org/fr/usagers/politique-dutilisation/ 


\section{Hors-thème}

François Vellas*

\section{Tourisme et économie internationale}

Le tourisme international représente, au même titre que le commerce international, une partie des echanges economiques mondiaux qui constitue le domaine de l'économic intemationale.

Toutefois, le tourisme intemational a été longtemps absent du champ danalyse des théories ćconomiques internationales et cela alors que de nombreuses études ont été consacrées à l'économie internationale des services notamment bancaires et de transport. Il en était ainsi parce que le tourisme international n'était pas considéré par les économistes comme une activité spécifique susceptible de justifier des études théoriques approfondies. Et parce quil ne représentait qu'une part relativement faible des recettes d'exportations de l'ordre de $5 \%$ à $6 \%$ au niveau mondial.

Actuellement, on constate une évolution rapide en faveur d'une prise de conscience de plus en pous pousséc du rôle économique du secteur touristique. Cette nouvelle attitude a commencé à se manifester parce que le developpement touristique a foumi à de nombreux pays des solutions efficaces aux problèmes posés par le chômage et par la nécessité d'une diversification des activités économiques.

Par ailleurs, progressivement, le tourisme international, au cours de sa croissance, est apparu comme un moyen permettant de fournir des solutions aux problèmes posés par le déficit de la balance des paiements d'un grand nombre d'États, notamment pour compenser les achats de biens d'équipements, de produits agricoles et industriels. Il en a été ainsi dans les pays nouvellement industrialisés qui ont pu réaliser les investissements industriẹ nécessaires à leurs mutations économiques grâce aux devises fournies par les entrées touristiques comme par exemple dans des pays aussi différents que l'Espagne, le Portugal, la Grece, la Yougoslavie en Europe; à Singapour. Hong-Kong et en Thailande dans le Sud-Est Asiatique.

Tel est aussi le cas de nombreux pays en développement parmi lesquels tout particulièrement des pays insulaires.

L'importance des flux du tourisme international pour la balance des paiements de nombreux pays conduit à se demander quels sont les déterminants de la spécialisation internationale dans lc tourisme. Question qui se situe au coeur de l'économie intemationale dont l'objet est d'étu- dier les causes fondamentales des echanges ćconomiques entre pays, de rechercher les déterminants théoriques de ces échanges, et par conséquent, les facteurs qui sont à la base de la specialisation internationale des pays.

Dans le domaine du tourisme international, la question fondamentale qui se pose est de savoir si les déterminants des théories du commerce international lui sont applicables ou s'il est nécessaire de rechercher d'autres déterminants gui lui seraicnt spécifiques.

Deux déterminants principaux seront analysés successivement:

- le déterminant de loffre avec les couts comparatifs et les dotations factoriclles:

le déterminant de la demande avec l'analyse de Linder.

\section{Tourisme international et déterminants de l'offre}

Depuis D. Ricardo, la théorie de l'avantage comparatif occupe une place essentielle dans l'analyse économique internationale. L'analyse des coûts comparatifs montre que chaque pays a intérêt à se spécialliser dans la production de biens ou de services dont le coût relatif de production est plus faible quà l'ćtranger. Cette difference de coûts comparatifs joue un rôle essentiel dans les produits touristiques. Elle peut permettre d'expliquer les différences de flux touristiques de pays à pays.

Cependant, l'étude des coûts comparatifs ne concerne pas seulement la comparaison internationalle de certains prix. Ils introduisent dans l'analyse des déterminants de l'échange international un facteur qualitatif, notamment le rapport qualité-prix qui joue un rôle essentiel dans la comparaison entre les produits touristiques.

\section{Les fondements théoriques de l'avantage comparatif}

La théorie de l'avantage comparatif permet de montrer que, même si un pays est en mesure de produire la totalité des biens el services qu"il consomme, et cela aux meilleurs couts, relatifs par rapport aux autres pays, il est cependant de son intérêt de se spécialiser dans les producitons les plus perfomantes et d'importer les autres biens. Dans ce cas, le pays va limiter volontairement sa production atux seuls biens et services dont le cout est relativement le plus faible comparé aux coûts à l'érranger. L'échange inter-
* Monsieur François Vellas est professeur d'économie internationale a l'Université de Toulouse I. 
national qui résulte de cette spécialisation lui permel ainsi daccroitre sa consommation globale et done de réaliser un gain grâce au commerce international.

Cette thónie des coûts comparalifs permet de foumir une explication pour un grand nombre d'echanges touristiques internationaux. Toutefois, la specialisation intemationale qui résulte de l'avantage comparatif n'est pas totale. L'analyse des échanges commerciaux internationaux indique seulement une tendance à la spécialisation en fonction des coûts comparatif $s^{(1)}$.

La thérie des couts eomparatifs permet de montrer que même si un pays est en mesure de produire la totalité des biens et services qu'il consomme, et cela aux meilleurs couts relatifs par rapport aux autres pays, il est cependant dans son interet de limiter sa production aux seuls biens et services dont le coût de la production est relativement le plus faible, compare aux coûts étrangers, en échangeant la part non consommée de ces biens contre ceux dont le coût de production est relativement plus élevé et qui sont produits â l'érranger'?

Cette theorie implique une specialisation internationale des pays dans la production et l'exportation de biens et services pour lesquels ils sont les plus performants. Cependant, cette spécialisation est rarement totale. L'analyse des êchanges commercian internationaux indique seulement une tendance à la spécialisation en fonction des coûts comparatifs. Il en est de mème dans le domaine du tourisme international où. malgré des différences importantes de coûts, la spécialisation de pays, par types de produit touristique, demeure difficile à déterminer à partir des seules différenoes de prix. Cependant, les coûts comparatifs peuvent être considérés comme un des facteurs déterminants de la répartition ef de l'évolution du tourisme international.

Le modèle des coûts comparatifs considéré à partir de lexemple même de Ricardo (Portugal, Grande-Bretagne) permet d'expliquer les échanges bilateraux de produits touristiques à partir des différences de coutts comparatifs.

Soit: 2 pays: pays (M) pays (P)

1 facteur: le travail (L) disponible en qualité égale dans les deux pays.

2 produits: produit (1) et produit (2).

Il est possible de représenter les conditions de production dans le pays (M) et dans le pays (P).

$\mathrm{L}_{1}^{\mathrm{M}}, \mathrm{L}_{1}^{\mathrm{p}}, \mathrm{L}_{2}^{\mathrm{M}}, \mathrm{L}_{2}^{\mathrm{P}}$ representent les quantites produites de biens $(1,2)$ dans le pays ( $M, P)$.

$\mathrm{X}_{1}^{\mathrm{M}}, \mathrm{X}_{1}^{\mathrm{P}}, \mathrm{X}_{2}^{\mathrm{M}}, \mathrm{X}_{2}^{\mathrm{P}}$ représentent les quantites produites de biens $(1,2)$ dans le pays (M, P).
$\mathrm{fl}_{1}^{\mathrm{M}} \mathrm{I}_{1}^{\mathrm{P}} \mathrm{f}_{2}^{\mathrm{M}}+\mathrm{f}_{2}^{\mathrm{P}}$

$\mathrm{T}^{\mathrm{M}}, \mathrm{T}^{\mathrm{M}}, \mathrm{T}^{\mathrm{T}^{2}}, \mathrm{~T}^{\mathrm{F}^{\mathrm{p}}}$ représentent les frontières de possibilités de production des pays (M, P).

C'est à partir de la comparaison des techniques de production dans chaque pays pour chaque bien qu'il est possible de déterminer l'avantage relatif de chacun des deux pays.

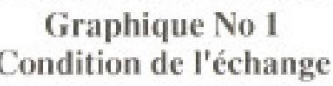

I

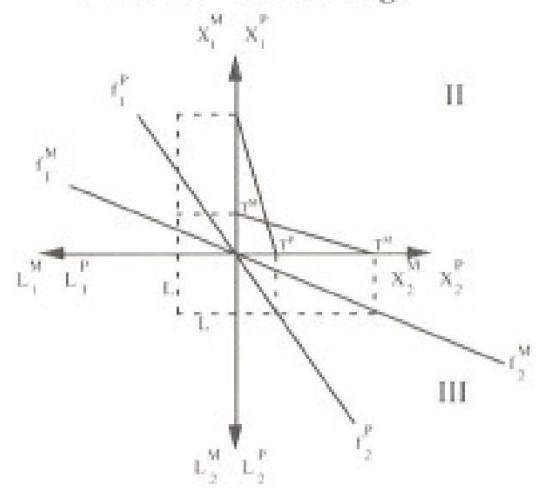

Ainsi, dans le schema précédent, les pays (M) ont un avantage comparatif dans la production du bien (2) et le pays ( $P$ ) détient un avantage comparatif dans la production du bien $(1)$.

Il cr est de mểme dans le domaine du tourisme international oủ, malgré des différences importantes de coutt, la specialisation des pays, par types de produit touristique, est difficile à déterminer à partir des seules differences de prix. Les coûts comparatifs n'ont pas moins une influence considérable ầ la fois sur l'évolution des flux du tourisme international et sur leur répartition de pays à pays.

\section{L'avantage comparatif touristi- que}

Parmi les déeminants du tourisme intemational. les coûts comparatifs joucnt un rôle essentiel. Leur influence dépend des spécificités du tourisme intemational. En raison de la très grande diversité des produits touristiques proposes, les comparaisons de coûts entre des produits Équivalents est souvent difficile si ce n'est impossiblẹ à réaliser dans les conditions strictes du modele de Ricardo.

Cependant, les produits touristiques (1) et (2) sont des services dont la production ne peut être répétée de façon identique. Il en résulte que l'étude de l'influence des coûts comparatifs doit être adaptée aux spécificités du tourisme international. En particulier, elle ne se réduit pas à la seule analyse des composantes du coût (transport, hébergement, réceptifs), mais elle conceme également le rapport qualitế-prix et l'ensemble des prestations annexes.

\section{Le rapport qualité-prix}

La comparaison internationale du rapport qualité-prix des produits touristiques de pays à pays permet dexpliquer une partie importante de la répartition et de levolution des flux du tourisme intemational. La notion de qualite importe souvent plus que le prix. II résulte que des pays tels que la Suisse ou l'Allemagne Féderalle sont toujours aux premiers rangs des pays touristiques du monde bien que les prix touristiques y soient plus élevés quailleurs. Dians ces conditions, comme l'indique le rapport Trigano(1984). "l'axe de développement à privilégier reste la qualité du service. Cest le meilleur moyen de se protéger de la concurrence internationale. Et cest le meilleur avantage comparatif de la France!",

Ce rapport qualité-prix pennet au secteur du tourisme d"un pays de resserrer les contraintes de la concurrence étrangère en pratiquant une politique de differrenciation basee sur l'élévation de la qualité des produits et des prestations touristiques.

\section{L'innovation et le progrès technologique}

L'innovation représente le second volet des politiques de différenciation qui ont pour but de renforcer les avantages comparatifs dont un pays dispose. Avec la recherehe du meilleur niveau de quallité, l'innovation permet d'assurer une meilleure spécialisation internationale du pays à long terme. Pour la plupart des pays développés, il est de plus en plus difficile de produife des prestations touristiques banalisees du type forfait hôtel-restaurant-plage à un prix compétitif, Jusqu'à présent, l'amélioration de la qualité a permis de réussir des politiques de differenciation et de pratiquer des prix relativement élevés. Cependant, des progrès considérables sont accomplis dans de nombreux pays qui ont fixé l'amélioration de la qualité comme objectif primordial de leurs politiques touristiques, Laide des Organisations Internationales, principalement du B.I.T. et l'O.M.T. s'est révêlee primordiale pour atteindre ces objectifs notamment pour la formation professionnelle avec la création du Centre International de Perfectionnement Professionnel et Technique de Turin.

Dans ces condifions, les pays développés, pour maintenir ou pour accroitre leurs avantages comparatifs dans le tourisme international ont recours, comme c'est le cas dans l'industrie, à des politiques d'innovation et de progrès technique.

\section{Le coût du transport}

L'évolution dụ coût dụ transport a une influence déterminante sur le coût des produits touristiques. Le cout du transport est fonction de divers tacteurs, notamment de la technologie (adapta- 
tion des moyens de transport aux besoins du tourisme international), de la structure des entreprises de transport, du prix des carburants et de la politique des Etats. En particulier, les politiques specifiques en faveur de l'abaissement des coûls du transport peuvent avoir un effet déterminant sur le tourisme international. N'a-t-on pas souligné "la prodigicuse élasticité du marché par rapport aux prix!".

Pour le transport routier, ce sont par exemple les "bons d'essence" ou des réductions sur les pćages d'autoroutes consenties aux visiteurs ctrangers (Italie, Yougoslavie, Roumanic):

Pour letransport ferroviaire, ce sont les billets touristiques à tarif reduit (France, Italie, R.F.A., Canada);

Pour le transport aérien, les accords d'abaissement tarifaire sur les vols réguliers et la libéralisation des vols charters jouent un rồle déterminant dans la constitution des flus touristiques vers les destinations qui ne peuvent pas être desservies par les transports terrestres.

\section{Le coût de l'hébergement}

Le coût de l'hébergement influence directement le mouvenent touristique, surtout en periode de crise économiques. En effet, le prix du transport a dû̉ ctre souvent "compensé" par des contraintes dans le prix de l'hébergement afind'offrir aux touristes un produit compose correspondant à leurs possibilités d'achat, c'est-à-dire à leurs demandes.

Les cot̂tls de l'hêbergement sont fortement influences par divers facteurs:

l'adoption de technologies nouvelles dans lat construction et dans les équipements; tel est le cas, par exemple, des nouvelles chaînes d'hôtels deux étoiles qui, en utilisant des techniques de constructions nouvelles, en innovant dans les formules d'accueil et de services à la clientèle ont pu apporter une amélioration du service tout en parvenant à une diminution du coût de l'hebergement.

les dépenses de personnel, les gains de productivite jouent un róle considérable dans lat comparaison internationale du coût d'hébergement; grấce aux nouvelles techniques utilisées, certains pays développés connaissent un "retour d'avantages comparatif's" malgro des taux de salaires plus alevés, et redeviennent competitils par rapport aux pays à bas salaires. Ce phénomène, déjà observế dans l'industrie textile (B. Ducros, 1983), s'explique par l'accéleration du progrés technique.

\section{Les coûts des services touristi- ques}

Les coûts des services touristiques autres que ceux de transport et d'hébergement sont relatifs, principalement à l'accueil, à la restauration, à des loisirs divers. Ces coûts sont difficilement comparables de pays à pays, parce qu'ils concernent des prestations touristiques extrềmement héterogènes. Mais it est nécessaire d'en tenir compte parce qu'ils contribuent, aussi, à expliquer la répartition et l'évolution des flux touristiques internationaux.

\section{Tourisme international et théorie des dotations factoriel- les}

Les dotations factorielles peuvent être considérées comme le déterminant principal du tourisme international particulièrement lorsque les ressources naturelles jouent un rôle essentiel. Les dotations factorielles sont détermintes par l'importance des ressources (facteurs) dont dispose un pays pour assurer la production de l'ensemble des services de tourisme international. Labondance relative de ces ressources a une influence décisive pour expliquer la place d'un pays dans le tourisme international.

Les dotations factorielles peuvent être rêparties. en trois catégories principales:

ressourecs naturelles, patrimoine historique, artistique et culturel:

ressourees humaines en travail et en qualification;

- ressources en capital et en infrastructure.

Les différences en dotations factoriclles sont souvent à l'origine de differences de coûts comparatifs. Cependant, elles ne sont pas sulfisantes pour expliquer l'ensemble des flux du tourisme international qui dépendent également des contraintes, de la (des) demande(s).

\section{Tourisme international et déterminants de la demande représentative}

L'importance du facteur "demande" a été mis en evidence dans lanalyse économique intemationale par Linder en 1960.

Les théories de la demande permettent dexpliquer à la fois les raisons du développement des flux touristiques et lintensité des courants dechanges touristiques entre pays.

La demande touristique représente les quantites de biens et de services touristiques que les consommatcurs desirent se procurer à un moment donné. Cette demande est directement fonction des niveaux de revenu par habitant, de l'intérêt d'une population pour le tourisme intemational (par exemple tres élevée pour le Canada, plus fáble pour la Norvecge), de la réglementation en vigueur (facilitations) concernant par exemple les passeports el visas, les sorties de devises, etc...

Un indicateur de la demande touristique intemationale peut etre fourni par l'evolution du nombrẹ de séjour's de vacances et par le pourcentage de séjours à létranger dans les scejours totaux.

La theorie de la demande a pour but dexpliquer la specialisation internationale des pays a partir des conditions de la dematide intérieure, régionale et internationale. Cette explication théorique paraît particulièrement bien adaptéc à licr lâ répartition internationale des flux touristiques at l'importance relative du tourisme international dans l'economie des pays. L'importance relative de la demande permet en effet de comprendre certains caracteres essentiels de Tevolution du tourisme international, notamment lia concentration des échanges touristiques entre pays semblables mais differencies et de niveau de développement économique êlevé.

Cette thérie de la demande énoncée des 1961 par Linder complete les explications theoriques par les dotations factorielles et par les coutts comparatifs.

\section{L'analyse de Linder}

Linder remarque que la seule difference dans les proportions factorielles n'est pas toujours determinante dans lexplication des échanges internationaux, En effel, les échanges les plus intensifs s'effectuent te plus souvent entre les economie présentant des caractéristiques proches du point de vue des dotations factorielles. Tel est le cas dans le tourisme intemational ou les échanges touristiques les plus importants concernent des pays voisins, principalement en Europe:

Dans ces conditions, Linder rejette, en grande partic, lat théorie des dotations factorielles et construit une nouvelle théoric de l'échange international, à partir de la demande intérieure ou demande représentative.

Pour Linder, la spécialisation internationale d'un pays dépend de l'existence d'une demande domestique suffisamment importante. Tel est le cas, semble-t-il, pour les principaux pays rócepteurs de touristes internationaux. Pour la plupart dentre eux, la demande touristique intemationale complète une demande touristique nationale importante. Cette forte demande intérieure crue les conditions favorables au developpement du tourisme international. Un savoir-faire, une technologie, un environnement propice viennent compléter les infrastructures qui confêrent au pays un avantage comparatif détermiกant.

Ainsi, le volume du tourisme international sera d'autant plus importantentre partenaire queceuxci auront une structure similaire de leur de- 
mande intéricure. Est-ce à dire que plus les pays sont semblables, plus leurs echanges touristiques sont importants? La réponse à cente question est donné par un prolongement théorique de lanalyse de la demande de B. LassudricDuchẻne (1971) qui montrequ'en fait, la base de l'échange international est avant tout une "demande de differences" cntre les partenaires à l'echange.

\section{La demande de différence}

L'échange international dépend à la fois du volume de la demande et de la différenciation des produits. Méme si les produits cehanges sont semblables, il existe toujours une difterence de qualite, ou de marque qui provoque un échange intemational. Cette "demande de différence" est particulièrement déterminante dans le tourisme intemational. Elle sappuie sur des differrences d'environnement géographique, culturel, linguistique, qui font l'objet d'echanges entre pays voisins de niveaux de développement cconomique comparable.

Dés lors, deux pays géographiquement proches, caractérisés par des dotations de facteurs peu differentes, pourront échanger des flux touristiques importants parce que des differences de marques, de prix, de qualité subsistent provoquant des flux que la proximité géographique favorise par ailleurs. D'autant plus que dans deux pays ayant des dotations de facteurs semblables, les goûts des consommatteurs peuvent être differents.

Tandis que des pays ayant des dotations factorielles très différentes peuvent n'échanger que des flux touristiques beaucoup plus faibles en raison de l'éloignement géographique de leurs demandes respectives et des gouts des consommatcurs.

Ainsi, la demande permet d'expliquer non seulement les causes du tourisme international mais surtout leur intensité. Le wolume des échanges de tourisme international sera d'autant plus important entre partenaires que ceux-ci auront une demande touristique intérieure élevếe et à structure similaire. Il en résulte que le tourisme international se développe particulièrement dans des zones géfographiques qui regroupent des pays industrialisés, caractérisés par ailleurs par un haut pouwoir d'achat, et d'autant plus qu'ils sont peu éloignés.

Le röle de la demande dans le tourisme international peut être mesuré par une êvaluation quantitative de la demande interieure et de la demande internationale, notamment à partir des statistiques de l'O.M.T.-

\section{Tourisme international et cycle de vie des produits}

Une nouvelle étape peut être franchie dans l'analyse economique du tourisme international avec la prise en comple du cycle de vie des produits.

La thếtie du cycle de vie du produit appliquêe au commerce international par Vernon en 1966 est aujourd hui considérée comme un des déterminants les plus performants de la spécialisation internationale.

En combinant lanalyse de la firme et l'analyse de l'économie internationale, la théorie du cycle de vie du produit permet de montrer comment évolue la spécialisation internationale.

Cette analyse peut-elle stappliquer à un tourisme international?

Pour répondre a cette question, il convient de prendre en compte le facteur technologique qui est à la buise du processus de cycle de vie du produit industricl. $\mathrm{Or}_{+}$on constate que le facteur technologique est de plus en plus important dans. les produits touristiques.

L'innovation touristique permet d'expliquer le transfert d'activité touristique de pays à pays tel qu'il s'effectue par exemple dans le domaine des. Parcs de Loisirs.

- Lepays leader réalise l'innovation(parexemple les U.S.A. avec Disney World) grâce bu un avantage technologique ef à une forte demande intérieure. La création de ce produit touristique nouveau provoque l'arrivee de flux importants de touristes intemationaux (canadiens, sud-américains et européens à Orlando).

- Le pays développé intermédiatire importe la technologie pour créer un nouweau produit touristique qui répond à sa demande intérieure et à la denande régionale des autres pays (implantation de l'Eurodisneyland a Marne-la-Vallec). Il y a transfert technologique du pays leader vers le pays intermédiaire et création d'un nouveau produit touristique à vocation régionale, nationale et européenne.

- Les pays en retard ne pourront espérer développer ces nouveaux produits touristiques que lorsque, la technologie étant banalisée. ils pourront bénéficier d'un avantage comparatif grâce à des couts de production de ce produit qui seront beaucoup plus bas.

Il apparant ainsi que les procédés d'analyse de l'économie internationale peuvent être appliqués au tourisme international.

Ils permettent de mieux comprendre et des lors de prévoir.

* Comprendre pourquoi un pays peut reussir ou échouer dans sa politique de développement du tourisme international.

* Prévoir dans une large mesure, l'ćvolution des flux touristiques des stratégies des opérateurs et des produits touristiques.

\section{NOTES ET RÉFÉRENCES}

(1) Cete analye de l'echunge intematimal a ce applinuee par Ricando an Commerec Intemational des 1817 .

(2) J-L. Muchielli, Principes d'B̈cunomies InternationaIEs. Econonica, Paris, 1488.

(3) S.-B. Linder, An Esay on Trade and Transforman tion, John Willey and Sons, New York, 1461.

CAZES, B., Le tourisme interoational, Hathic, Paris, 1989

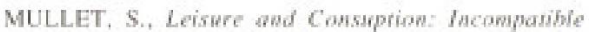
Cowcens, Leisure Studies, vol, 7 , no 3 septembre 1988

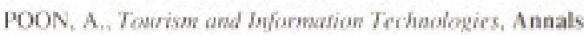
of Tourism Research. wol 15, nle 4, 1988.

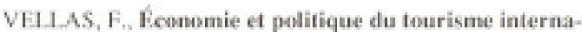
tional, Ecomentrica, IOSS, 